\title{
SONICATED ASSISTED SYNTHESIS OF BENZIMIDAZOLES, BENZOXAZOLES AND BENZOTHIAZOLES IN AQUEOUS MEDIA
}

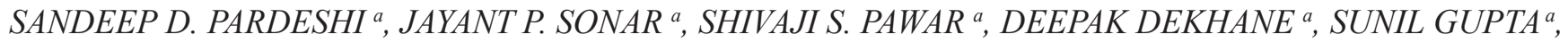 \\ ASHOK M. ZINE ${ }^{a}$, SHIVAJI N. THORE ${ }^{b, *}$
}

${ }^{a}$ Department of Chemistry, Vinayakrao Patil Mahavidyalaya, Vaijapur, Maharashtra, India. 423701
${ }^{b}$ Department of Chemistry, Deogiri College, Aurangabad, Maharashtra, India.431005

(Received: June 4, 2013 - Accepted: December 8, 2013)

\begin{abstract}
Ammonium nickel sulphate $\left[\left(\mathrm{NH}_{4}\right)_{2} \mathrm{SO}_{4} \cdot \mathrm{NiSO}_{4} \cdot 6 \mathrm{H}_{2} \mathrm{O}\right]$ was found as a new catalyst to synthesis 2-aryl benzimidazole, 2-aryl benzothiazole and 2-aryl benzoxazole in aqueous media under sonication irradiation. The procedure is an eco-friendly, efficient and provides simple workup and good yield.
\end{abstract}

Keywords: - Benzimidazole, benzothiazole, benzoxazole, aqueous media, sonication, ammonium nickel sulphate.

\section{INTRODUCTION}

The benzimidazoles, benzoxazole and benzthaizoles are an important heterocyclic nucleus which has been widely used in medicinal chemistry ${ }^{1,2}$. These heterocycles are an important pharmacophore ${ }^{3,4}$ in drug discovery and good intermediate ${ }^{5}$ in synthesis of many important organic compounds. These heterocycles shows different pharmacological properties such as antibacterial ${ }^{6}$, antiviral ${ }^{7}$, antifugal ${ }^{8}$, anticancer ${ }^{9}$, anticonvulsant ${ }^{10}$ and immunosuppressant 11.

These heterocycles can be prepared by condensing carboxylic acid ${ }^{12}$, acid chloride 13, 14, orthoester ${ }^{15-17}$, esters ${ }^{18}$ and aldehydes ${ }^{19-24}$ with o-phenylenediamine, o-aminophenols and o-aminothaiophenols, dehydration of o-acylaminophenols ${ }^{25}$, reaction of o-quinones with amines ${ }^{26}$ and Beckmann rearrangement of $\mathrm{o}$-acylphenoloximes ${ }^{27}$. The most common method of synthesis of these heterocycles includes condensation of o-phenylenediamine, o-aminophenol or o-aminothiophenol with suitable aldehyde ${ }^{28-32}$. Most of these procedure have their own advantages and disadvantages, thus there is still a need to search better ecofriendly procedure.

The toxic and volatile natures of many organic solvents have posed serious environmental problems. Due to this organic reaction in aqueous media have attracted much attention in synthetic organic chemistry because water is one of the most abundant, cheap and environmental friendly solvent however there are very few reports for synthesis of 1,3 benzazoles in aqueous media.

Ultrasound irradiation has been established as an important technique in synthetic organic chemistry. It has been used as an efficient energy source for the organic reactions. Simple experimental procedure, very high yields, increased selectivity and clean reaction of many ultrasound induced organic transformations offers additional convenience in the field of synthetic organic chemistry ${ }^{33-36}$. These finding promotes us to investigate the synthesis of benzimidazoles, benzoxazole and benzothiazoles in aqueous media.

\section{EXPERIMENTAL}

Bandelin Sonorex $(35 \mathrm{kHz})$ ultrasonic bath was used for ultrasonic irradiation. ${ }^{1} \mathrm{H}$ NMR spectra were recorded on Mercury Plus Varian in DMSO at $400 \mathrm{MHz}$ using TMS as an internal standard. Mass spectra were recorded on Micromass Quattro II using electrospray Ionization technique, showing $(\mathrm{M}+\mathrm{H})$ peak as a base peak. The progress of the reactions was monitored by TLC (silica, 80:20 hexane/ ethyl acetate).

\section{General Procedure for the Preparation of 3a-31}

o-phenylene diamine $(1 \mathrm{mmol})$, aromatic aldehyde $(1.1 \mathrm{mmol})$ and water $(10 \mathrm{~mL})$ were mixed in $25 \mathrm{~mL}$ single neck round bottom flask, and to this Ammonium Nickel Sulphate (10 mol \%) was added. The reaction mixture was sonicated at room temperature $\left(25^{\circ} \mathrm{C}\right)$ for the appropriate time (Table 2, entries $1-12$ ), and the progress of reaction was monitored by TLC. After completion of reaction, the mixture was extracted with ethyl acetate $(2 \times 10 \mathrm{~mL})$. The combined organic layer was dried over anhydrous $\mathrm{Na}_{2} \mathrm{SO}_{4}$ and evaporated under reduced pressure; the crude material was purified by column chromatography over silica gel to afford products $3 \mathrm{a}-31$ with high purity.
Selected spectral data

2-phenyl-1H-benzimidazole $\mathbf{3 a}$

${ }^{1} \mathrm{H}$ NMR(400MHz DMSO) $\delta 12.7$ (s, 1H, NH), 7.95(m, 2H, ArH), 7.257.35(m, 5H, ArH), 7.05(2H, ArH); $m / z$ 195(M+H). Elemental analysis Calcd. for $\mathrm{C}_{13} \mathrm{H}_{10} \mathrm{~N}_{2}$. C, 80.39; H, 5.19; N, 14.42. Found: C, 80.42; H, 5.17; N, 14.41.

2-(4-methoxy phenyl)-1H-benzimidazole $\mathbf{3 b}$

${ }^{1} \mathrm{H}$ NMR(400MHz DMSO) $\delta 3.8(\mathrm{~s}, 3 \mathrm{H}, \mathrm{OCH} 3), 7.09-7.11(\mathrm{~d}, 2 \mathrm{H}, J 9.2 \mathrm{~Hz}$, $\mathrm{ArH}), 7.16-7.18(\mathrm{~m}, 2 \mathrm{H}, \mathrm{ArH}), 7.53-7.56(\mathrm{~m}, 2 \mathrm{H}, \mathrm{ArH}), 8.08-8.11(\mathrm{~d}, 2 \mathrm{H}, J$ $8.8 \mathrm{~Hz}, \mathrm{ArH}) ; \mathrm{m} / \mathrm{z} 225(\mathrm{M}+\mathrm{H})$. Elemental analysis Calcd. for $\mathrm{C}_{14} \mathrm{H}_{12} \mathrm{~N}_{2} \mathrm{O}$. C, 74.98; H, 5.39; N, 12.49. Found: C, 75.03; H, 5.36; N, 12.45 .

2-(4-chlorophenyl)-1H-benzimidazole $\mathbf{3 c}$

${ }^{1} \mathrm{H}$ NMR(400MHz DMSO) $\delta 12.5(\mathrm{~s}, 1 \mathrm{H}, \mathrm{NH}), 8.20(\mathrm{~d}, 2 \mathrm{H}, \mathrm{ArH}), 7.6$ (d, 2H, ArH), 7.3 (m, 2H, ArH), 7.1 (m, 2H, ArH); m/z 229(M+H). Elemental analysis Calcd. for $\mathrm{C}_{13} \mathrm{H}_{9} \mathrm{~N}_{2} \mathrm{Cl}$. C, 68.28; H, 3.97; N, 12.25. Found: C, 68.20; $\mathrm{H}, 4.01 ; \mathrm{N}, 12.28$.

5-Chloro-2-(4-methoxyphenyl)-1H-benzimidazole 3d

${ }^{1} \mathrm{H}$ NMR(400MHz DMSO) $\delta 7.97(\mathrm{~d}, J 9.2 \mathrm{~Hz}, 2 \mathrm{H}), 7.86(\mathrm{~s}, 1 \mathrm{H}), 7.67(\mathrm{~d}$, $J 8.1 \mathrm{~Hz}, 1 \mathrm{H}), 7.35(\mathrm{~d}, J 8.1 \mathrm{~Hz}, 1 \mathrm{H}), 6.92(\mathrm{~d}, J 9.2 \mathrm{~Hz}, 2 \mathrm{H}), 3.75(\mathrm{~s}, 3 \mathrm{H}) ; \mathrm{m} / z$ $276(\mathrm{M}+\mathrm{H})$. Elemental analysis Calcd. for $\mathrm{C}_{14} \mathrm{H}_{11} \mathrm{ClN}_{2} \mathrm{O} . \mathrm{C}, 65.00 ; \mathrm{H}, 4.29 ; \mathrm{N}$, 10.83. Found: $\mathrm{C}, 65.05 ; \mathrm{H}, 4.25 ; \mathrm{N}, 10.80$.

5-Chloro-2-(4-nitrophenyl)-1H-benzimidazole $\mathbf{3 e}$

${ }^{1} \mathrm{H}$ NMR(400MHz DMSO- $\left.d_{6}\right) \delta 8.52(\mathrm{~d}, J 9.4 \mathrm{~Hz}, 2 \mathrm{H}), 7.96(\mathrm{~d}, J 9.4$ $\mathrm{Hz}, 2 \mathrm{H}), 7.85(\mathrm{~s}, 1 \mathrm{H}), 7.71(\mathrm{~d}, J 8.2 \mathrm{~Hz}, 1 \mathrm{H}), 7.32(\mathrm{~d}, J 8.2 \mathrm{~Hz}, 1 \mathrm{H}) ; \mathrm{m} / z 274$ $(\mathrm{M}+\mathrm{H})$. Elemental analysis Calcd. for $\mathrm{C}_{13} \mathrm{H}_{8} \mathrm{ClN}_{3} \mathrm{O}_{2} . \mathrm{C}, 57.05 ; \mathrm{H}, 2.95 ; \mathrm{N}$, 15.35. Found: C, 56.98; H, 3.01; N, 15.30.

5-Chloro-2-(4-N,N-dimethylaminophenyl)-1H-benzimidazole: $\mathbf{3 f}$ ${ }^{1} \mathrm{H}$ NMR(400MHz DMSO- $\left.d_{6}\right) \delta 7.94(\mathrm{~d}, J 8.9 \mathrm{~Hz}, 2 \mathrm{H}), 7.85(\mathrm{~s}, 1 \mathrm{H}), 7.66$ $(\mathrm{d}, J 8.3 \mathrm{~Hz}, 1 \mathrm{H}), 7.37(\mathrm{~d}, J 8.3 \mathrm{~Hz}, 1 \mathrm{H}), 6.82(\mathrm{~d}, J 8.9 \mathrm{~Hz}, 2 \mathrm{H}), 3.35(\mathrm{~s}, 6 \mathrm{H}) ;$ $\mathrm{m} / z 272(\mathrm{M}+\mathrm{H})$. Elemental analysis Calcd. for $\mathrm{C}_{15} \mathrm{H}_{14} \mathrm{ClN}_{3}$. C, 66.30; H, 5.19; $\mathrm{N}, 15.46$. Found: C, 66.38; H, 5.12; N, 15.40 .

2-(3-nitrophenyl)-1H- benzimidazole $\mathbf{3 g}$

${ }^{1} \mathrm{H}$ NMR(400MHz DMSO- $d$ ) $\delta 12.9(\mathrm{~s}, 1 \mathrm{H} \mathrm{NH}), 8.90(\mathrm{~s}, 1 \mathrm{H} \mathrm{Ar} \mathrm{H}), 8.50$ $(\mathrm{d}, 1 \mathrm{H} \mathrm{Ar} \mathrm{H}), 8.10$ (d,1H Ar H), $7.70(\mathrm{t}, 1 \mathrm{H} \mathrm{Ar} \mathrm{H}), 7.50$ (m, 2H ArH), $7.2(\mathrm{~m}$, $2 \mathrm{H} \mathrm{ArH}) ; \mathrm{m} / z 240(\mathrm{M}+\mathrm{H})$. Elemental analysis Calcd. for $\mathrm{C}_{13} \mathrm{H}_{9} \mathrm{~N}_{3} \mathrm{O}_{2}$. C, 65.27; H, 3.79; N, 17.56. Found: C, 65.32; H, 3.70; N, 17.62.

2-pyridin-3yl-1H-benzimidazole $\mathbf{3 h}$

${ }^{1} \mathrm{H}$ NMR $\left(400 \mathrm{MHz}\right.$ DMSO- $\left.d_{6}\right) \delta 13.05(\mathrm{~s}, 1 \mathrm{H} \mathrm{NH}), 9.35(\mathrm{~d}, 1 \mathrm{H} \mathrm{ArH})$, 8.75(m,1H ArH), 8.60(m,1H ArH), 7.70(m,3H ArH) $7.40(\mathrm{~m}, 2 \mathrm{H} \mathrm{ArH}) ; m / z$ 196(M+H). Elemental analysis Calcd. for $\mathrm{C}_{12} \mathrm{H}_{9} \mathrm{~N}_{3} . \mathrm{C}, 73.83 ; \mathrm{H}, 4.65 ; \mathrm{N}, 21.52$. Found: C, 73.90; H, 4.60; N, 21.50 .

2-(2,3-Dimethoxyphenyl)-1H-benzimidazole $\mathbf{3 i}$

${ }^{1} \mathrm{H}$ NMR(400MHz DMSO- $\left.d_{6}\right) \delta 12.19(\mathrm{~s}, 1 \mathrm{H}, \mathrm{NH}), 7.84(\mathrm{dd}, 1 \mathrm{H}, J$ 
$7.3 \mathrm{~Hz}, 1.1 \mathrm{~Hz}, \mathrm{ArH}), 7.64$ (m, 3H, ArH), 7.20 (m, 3H, ArH), 3.90 (s, 3H, OCH3), $3.85(\mathrm{~s}, 3 \mathrm{H}, \mathrm{OCH} 3) ; \mathrm{m} / \mathrm{z} 255(\mathrm{M}+\mathrm{H})$. Elemental analysis Calcd. for $\mathrm{C}_{15} \mathrm{H}_{14} \mathrm{~N}_{2} \mathrm{O}_{2}$. C, 70.85; H, 5.55; N, 11.02. Found: C, 70.89; H, 5.49; N, 11.07.

\section{2-(3-Fluorophenyl)-1H-benzimidazole $\mathbf{3 j}$}

${ }^{1} \mathrm{H}$ NMR(400MHz DMSO- $d$ ) $\delta 13.00(\mathrm{~s}, 1 \mathrm{H}, \mathrm{NH}), 8.02(\mathrm{~d}, 1 \mathrm{H}, J 7.7 \mathrm{~Hz}$, ArH), $7.96(\mathrm{~m}, 1 \mathrm{H}, \mathrm{ArH}), 7.69(\mathrm{~m}, 1 \mathrm{H}, \mathrm{ArH}), 7.59(\mathrm{~m}, 2 \mathrm{H}, \mathrm{ArH}), 7.34(\mathrm{~m}$ $1 \mathrm{H}, \mathrm{ArH}), 7.23(\mathrm{~m}, 2 \mathrm{H}, \mathrm{ArH}) ; \mathrm{m} / z 213(\mathrm{M}+\mathrm{H})$. Elemental analysis Calcd. for $\mathrm{C}_{13} \mathrm{H}_{9} \mathrm{FN}_{2}$. C, 73.57; H, 4.27; N, 13.20. Found: C, 73.51; H, 4.32; N, 13.28.

\section{2-(3-chloro phenyl)-1H-benzimidazole $\mathbf{3 k}$}

${ }^{1} \mathrm{H}$ NMR $\left(400 \mathrm{MHz}\right.$ DMSO- $\left.d_{6}\right) \delta 8.41(\mathrm{t}, 1 \mathrm{H}, J 1.2 \mathrm{~Hz}, 2.0 \mathrm{~Hz}, \mathrm{ArH}), 8.20-$ 8.29(dd, $1 \mathrm{H}, J 1.2,7.6 \mathrm{~Hz}, \mathrm{ArH}), 7.80-7.84(\mathrm{~m}, 2 \mathrm{H}, \mathrm{ArH}), 7.69-7.77(\mathrm{~m}, 2 \mathrm{H}$ $\mathrm{ArH}), 7.50-7.54(\mathrm{~m}, 2 \mathrm{H}, \mathrm{ArH}) ; \mathrm{m} / z 229(\mathrm{M}+\mathrm{H})$. Elemental analysis Calcd. for $\mathrm{C}_{13} \mathrm{H}_{9} \mathrm{ClN}_{2}$. C, 68.28; H, 3.97; N, 12.25. Found: C, 68.35; H, 3.92; N, 12.20 .

\section{2-(4-nitrophenyl)-1H-benzimidazole 31}

${ }^{1} \mathrm{H}$ NMR(400MHz DMSO- $\left.d_{6}\right) \delta 8.37-8.39(\mathrm{~d}, 2 \mathrm{H}, J$ 8.4Hz, ArH), 8.14$8.17(\mathrm{~d}, 2 \mathrm{H}, J 8.8 \mathrm{~Hz}, \mathrm{ArH}), 7.70-7.72(\mathrm{~d}, 1 \mathrm{H}, J 7.6 \mathrm{~Hz}, \mathrm{ArH}), 7.65-7.67(\mathrm{~d}, 1 \mathrm{H}$, $J$ 8.4Hz, ArH) 7.32-7.35(m, 1H, ArH), 7.25-7.29(m,1H, ArH); $m / z 240(\mathrm{M}+\mathrm{H})$. Elemental analysis Calcd. for $\mathrm{C}_{13} \mathrm{H}_{9} \mathrm{~N}_{3} \mathrm{O}_{2}$. C, 65.27; H, 3.79; N, 17.56. Found: C, $65.20 ; \mathrm{H}, 3.87 ; \mathrm{N}, 17.50$

\section{General Procedure for the Preparation of $4 a-4 m$}

o-amino thiophenol $(1 \mathrm{mmol})$, aromatic aldehyde $(1.1 \mathrm{mmol})$ and water $(10 \mathrm{~mL})$ were mixed in $25 \mathrm{~mL}$ single neck round bottom flask, and to this Ammonium Nickel Sulphate $(10 \mathrm{~mol} \%)$ was added. The reaction mixture was sonicated at room temperature $\left(25^{\circ} \mathrm{C}\right)$ for the appropriate time (Table 2, entries 13-25), and the progress of reaction was monitored by TLC. After completion of reaction, the mixture was extracted with ethyl acetate $(2 \times 10 \mathrm{~mL})$. The combined organic layer was dried over anhydrous $\mathrm{Na}_{2} \mathrm{SO}_{4}$ and evaporated under reduced pressure; the crude material was purified by column chromatography over silica gel to afford products $4 \mathrm{a}-4 \mathrm{~m}$ with high purity.

Selected spectral data

2-Phenyl-1,3-benzothiazole 4a

${ }^{1} \mathrm{H}$ NMR(400MHz DMSO) $\delta 8.12(\mathrm{~d}, J 7.38 \mathrm{~Hz}, 1 \mathrm{H}, \mathrm{ArH}), 8.02(\mathrm{t}, J 7.42$ $\mathrm{Hz}, 1 \mathrm{H}, \mathrm{ArH}), 7.65(\mathrm{t}, J 7.42 \mathrm{~Hz}, 1 \mathrm{H}, \mathrm{ArH}), 7.33-7.39(\mathrm{~m}, 3 \mathrm{H}, \mathrm{ArH}), 7.19-$ 7.25(m, $3 \mathrm{H}, \mathrm{ArH}) . ; m / z 212(\mathrm{M}+\mathrm{H})$. Elemental analysis Calcd. for $\mathrm{C}_{13} \mathrm{H}_{9} \mathrm{NS}$. C, 73.90; H, 4.29; N, 6.63. Found: C, 73.82; H, 4.35; N, 6.58.

\section{2-(4-Methoxyphenyl)- 1,3-benzothiazole $\mathbf{4 b}$}

${ }^{1} \mathrm{H}$ NMR(400MHz DMSO) $\delta 8.19(\mathrm{~d}, J 8.22 \mathrm{~Hz}, 1 \mathrm{H}, \mathrm{ArH}), 8.05(\mathrm{~d}, J 8.22 \mathrm{~Hz}$ $1 \mathrm{H}, \mathrm{ArH}), 7.07(\mathrm{~d}, J 7.75 \mathrm{~Hz}, 2 \mathrm{H}, \mathrm{ArH}), 7.12-7.41(\mathrm{~m}, 4 \mathrm{H}, \mathrm{ArH}), 3.92(\mathrm{~s} 3 \mathrm{H}$, OCH3); $m / z 242(\mathrm{M}+\mathrm{H})$. Elemental analysis Calcd. for $\mathrm{C}_{14} \mathrm{H}_{11} \mathrm{NOS}$. C, 69.68 ; H, 4.59; N, 5.80. Found: C, 69.59; H, 4.65; N, 5.85.

\section{2-(4-Nitrophenyl) benzothiazole $\mathbf{4 c}$}

${ }^{1} \mathrm{H}$ NMR(400MHz DMSO- $\left.d_{f}\right) \delta 8.9$ (d, $\left.J 8.0 \mathrm{~Hz}, 2 \mathrm{H}, \mathrm{Ar}-\mathrm{H}\right) ; 8.32$ (d, $J$ $8.0 \mathrm{~Hz}, 2 \mathrm{H}, \mathrm{Ar}-\mathrm{H}) ; 8.23(\mathrm{~d}, J 8.0 \mathrm{~Hz}, 1 \mathrm{H}, \mathrm{Ar}-\mathrm{H}) ; 8.02(\mathrm{~d}, J 8.0 \mathrm{~Hz}, 1 \mathrm{H}, \mathrm{Ar}-$ $\mathrm{H})$; 7.44-7.53 (m, $2 \mathrm{H}, \mathrm{Ar}-\mathrm{H}) ; \mathrm{m} / z 256(\mathrm{M}+\mathrm{H})$. Elemental analysis Calcd. for $\mathrm{C}_{13} \mathrm{H}_{8} \mathrm{~N}_{2} \mathrm{O}_{2}$ S. C, 60.93; H, 3.15; N, 10.93. Found: C, 61.03; H, 3.20; N, 10.85 .

\section{2-Thienyl-1,3-benzothiazole $\mathbf{4 d}$}

${ }^{1} \mathrm{H}$ NMR(400MHz DMSO) $\delta 8.22(\mathrm{~d}, J 8.0 \mathrm{~Hz}, 1 \mathrm{H}, \mathrm{ArH}) ; 8.13(\mathrm{~d}, J 8.0$ $\mathrm{Hz}, 1 \mathrm{H}, \mathrm{ArH}) ; 7.74(\mathrm{~d}, J 4.0 \mathrm{~Hz}, 1 \mathrm{H}$, thiophene $\mathrm{CH}) ; 7.69(\mathrm{~d}, J 4.0 \mathrm{~Hz}, 1 \mathrm{H}$, thiophene $\mathrm{CH}) ; 7.54-7.63(\mathrm{~m}, 2 \mathrm{H}, \mathrm{Ar}-\mathrm{H}) ; 7.33(\mathrm{t}, J 4.0 \mathrm{~Hz}, 1 \mathrm{H}$, thiophene $\mathrm{CH})$; $\mathrm{m} / \mathrm{z} 217(\mathrm{M}+\mathrm{H})$. Elemental analysis Calcd. for $\mathrm{C}_{11} \mathrm{H}_{7} \mathrm{NS}_{2}$. C, 60.80; H, 3.25; N, 6.45. Found: C, $60.84 ; \mathrm{H}, 3.20 ; \mathrm{N}, 6.52$.

\section{2-(4-Fluoro phenyl) benzothiazole $4 \mathrm{e}$}

${ }^{1} \mathrm{H}$ NMR(400MHz DMSO- $\left.d_{6}\right) \delta 8.7$ (s, $1 \mathrm{H}$, pyrazolyl-H), $8.03(\mathrm{~d}, J 6 \mathrm{~Hz}$, $2 \mathrm{H}), 7.747 .85(\mathrm{~m}, 5 \mathrm{H}, \mathrm{Ar}-\mathrm{H}), 7.45-7.60(\mathrm{~m}, 5 \mathrm{H}, \mathrm{Ar}-\mathrm{H}), 7.31-7.38$ (dd, $J 8 \mathrm{~Hz}$, $6 \mathrm{~Hz}, 2 \mathrm{H}) ; \mathrm{m} / z 230(\mathrm{M}+\mathrm{H})$. Elemental analysis Calcd. for $\mathrm{C}_{13} \mathrm{H}_{8} \mathrm{FNS}$. C, 68.16; H, 3.52; N, 6.11. Found: C, 68.16; H, 3.45; N, 6.18.

\section{2-(4 methyl phenyl) benzothiazole $\mathbf{4 f}$}

${ }^{1} \mathrm{H}$ NMR(400MHz DMSO- $d$ ) $\delta 7.92-8.10(\mathrm{~m} 3 \mathrm{H} \mathrm{Ar} \mathrm{H}), 7.41(\mathrm{t} J 7.38 \mathrm{~Hz}$, $1 \mathrm{H} \mathrm{Ar} \mathrm{H}), 7.30$ (t $J 7.38 \mathrm{~Hz}, 1 \mathrm{H} \mathrm{Ar} \mathrm{H}), 7.26(\mathrm{~d} J 8.12 \mathrm{~Hz} 2 \mathrm{H} \mathrm{Ar} \mathrm{H}), 2.45(\mathrm{~s} 3 \mathrm{H}$ $\left.\mathrm{CH}_{3}\right) ; m / z 226(\mathrm{M}+\mathrm{H})$. Elemental analysis Calcd. for $\mathrm{C}_{14} \mathrm{H}_{11} \mathrm{NS}$. C, 74.63; H, $4.92 ; \mathrm{N}, 6.22$. Found: $\mathrm{C}, 74.69 ; \mathrm{H}, 4.88 ; \mathrm{N}, 6.28$.

\section{2-(4-cyanophenyl) benzothiazole $\mathbf{4 g}$}

${ }^{1} \mathrm{H}$ NMR(400MHz DMSO- $\left.d_{6}\right) \delta 8.09(\mathrm{~d}, J 8.15 \mathrm{~Hz}, 2 \mathrm{H}, \mathrm{ArH}), 8.05(\mathrm{dd}, J$
$2.42 \mathrm{~Hz}, 7.55 \mathrm{~Hz}, 2 \mathrm{H}, \mathrm{ArH}), 7.85(\mathrm{~d}, J 8.15 \mathrm{~Hz}, 2 \mathrm{H}, \mathrm{ArH}), 7.61(\mathrm{dd}, J 7.55 \mathrm{~Hz}$, $7.75 \mathrm{~Hz}, 2 \mathrm{H}, \mathrm{ArH}) ; m / z 237(\mathrm{M}+\mathrm{H})$. Elemental analysis Calcd. for $\mathrm{C}_{14} \mathrm{H}_{8} \mathrm{~N}_{2} \mathrm{~S} . \mathrm{C}$, 71.16; H, 3.41; N, 11.86. Found: C, 71.21; H, 3.44; N, 11.80 .

\section{2-(3,4,5 trimethoxy phenyl) benzothiazole $\mathbf{4 h}$}

${ }^{1} \mathrm{H}$ NMR $(400 \mathrm{MHz}$ DMSO- $d$ ) $\delta 8.31(\mathrm{t}, 1 \mathrm{H}, \mathrm{ArH}), 8.22(\mathrm{t}, 1 \mathrm{H}, \mathrm{ArH})$, $7.45(\mathrm{dd} J 2.42 \mathrm{~Hz}, 7.55 \mathrm{~Hz}, 2 \mathrm{H}, \mathrm{ArH}), 6.57(\mathrm{~d}, J 2.42 \mathrm{~Hz}, 2 \mathrm{H}, \mathrm{ArH}), 3.61(\mathrm{~s}, 9 \mathrm{H}$, $\mathrm{OCH} 3) . ; \mathrm{m} / z$ 302(M+H). Elemental analysis Calcd. for $\mathrm{C}_{16} \mathrm{H}_{15} \mathrm{NO}_{3} \mathrm{~S}$. C, 63.77; $\mathrm{H}, 5.02 ; \mathrm{N}, 4.65$. Found: C, 63.69; H, 4.99; N, 4.71.

\section{2-(4-chlorophenyl) benzothiazole $\mathbf{4 i}$}

${ }^{1} \mathrm{H}$ NMR(400MHz DMSO- $\left.d_{6}\right) \delta 8.15(\mathrm{~d}, J 7.3 \mathrm{~Hz}, 1 \mathrm{H}, \mathrm{ArH}), 8.05(\mathrm{~d}, J 8.4$ $\mathrm{Hz}, 2 \mathrm{H}, \mathrm{ArH}), 7.80(\mathrm{~d}, J 7.3 \mathrm{~Hz}, 1 \mathrm{H}, \mathrm{ArH}), 7.41(\mathrm{t}, J 7.6 \mathrm{~Hz}, 1 \mathrm{H}, \mathrm{ArH}), 7.30(\mathrm{t}$, $J 7.6 \mathrm{~Hz}, 1 \mathrm{H}, \mathrm{ArH}), 7.16(\mathrm{~d}, J 8.22 \mathrm{~Hz}, 2 \mathrm{H}, \mathrm{ArH}) ; \mathrm{m} / z 246(\mathrm{M}+\mathrm{H})$. Elemental analysis Calcd. for $\mathrm{C}_{13} \mathrm{H}_{8}$ ClNS. C, 63.54; H, 3.28; N, 5.70. Found: C, 63.61; $\mathrm{H}, 3.30 ; \mathrm{N}, 5.65$.

\section{6-Chloro-2-phenyl benzothiazole $\mathbf{4 j}$}

${ }^{1} \mathrm{H}$ NMR(400MHz DMSO- $\left.d_{6}\right) \delta 8.19(\mathrm{~d}, J 8.3 \mathrm{~Hz}, 1 \mathrm{H}, \mathrm{ArH}), 8.13(\mathrm{~d}, J$ $2.12 \mathrm{~Hz}, 1 \mathrm{H}, \mathrm{ArH}), 7.56(\mathrm{dd}, J 7.61 \mathrm{~Hz}, 2.17,2 \mathrm{H}, \mathrm{ArH}), 7.36(\mathrm{t}, J 7.61 \mathrm{~Hz}$ $2 \mathrm{H}, \mathrm{ArH}), 7.16(\mathrm{~m}, 1 \mathrm{H}, \mathrm{ArH}) ; \mathrm{m} / \mathrm{z} \quad 246(\mathrm{M}+\mathrm{H})$. Elemental analysis Calcd. for $\mathrm{C}_{13} \mathrm{H}_{8}$ ClNS. C, 63.54; H, 3.28; N, 5.70. Found: C, 63.49; H, 3.32; N, 5.78.

6-chloro-2(3-methoxy phenyl) benzothaizole $4 \mathbf{k}$

${ }^{1} \mathrm{H}$ NMR(400MHz DMSO- $\left.d_{6}\right) \delta 7.99(\mathrm{~d}, J 2.5 \mathrm{~Hz}, 1 \mathrm{H}, \mathrm{ArH}), 7.77(\mathrm{~d}, J$ $7.7 \mathrm{~Hz}, 1 \mathrm{H}, \mathrm{ArH}), \quad 7.60-7.70(\mathrm{~m}, 2 \mathrm{H}, \mathrm{ArH}), 7.32-7.50(\mathrm{~m}, 2 \mathrm{H}, \mathrm{ArH}), 7.16(\mathrm{dd}$ $J 2.5 \mathrm{~Hz}, 7.8 \mathrm{~Hz}, 1 \mathrm{H}, \mathrm{ArH}), 3.91(\mathrm{~s} 3 \mathrm{H}, \mathrm{OCH} 3) ; \mathrm{m} / z$ 276(M+H). Elemental analysis Calcd. for $\mathrm{C}_{14} \mathrm{H}_{10}$ CINOS. C, 60.98; H, 3.66; N, 5.08. Found: C, 61.05; $\mathrm{H}, 3.58 ; \mathrm{N}, 5.13$.

6-chloro-2-(4-chlorophenyl) benzothiazole 4I

${ }^{1} \mathrm{H}$ NMR(400MHz DMSO- $\left.d_{6}\right) \delta 8.17(\mathrm{~d}, J 7.9 \mathrm{~Hz}, 1 \mathrm{H}, \mathrm{ArH}), 8.13(\mathrm{~d}, J$ $2.2 \mathrm{~Hz}, 1 \mathrm{H}, \mathrm{ArH}), 7.56(\mathrm{dt}, J 7.9 \mathrm{~Hz}, 2.2 \mathrm{~Hz}, 1 \mathrm{H}, \mathrm{ArH}), 7.41(\mathrm{~d}, J 8.1 \mathrm{~Hz}, 2 \mathrm{H}$, $\mathrm{ArH}), 7.32(\mathrm{~d}, J 8.1 \mathrm{~Hz}, 2 \mathrm{H}, \mathrm{ArH}) ; \mathrm{m} / z 280(\mathrm{M}+\mathrm{H})$. Elemental analysis Calcd. for $\mathrm{C}_{13} \mathrm{H}_{7} \mathrm{Cl}_{2} \mathrm{NS}$. C, 55.73; H, 2.52; N, 5.00. Found: C, 55.70; H, 2.48; N, 5.06.

\section{2-(4-(piperidin-1-yl)phenyl)benzothiazole $\mathbf{4 m}$}

${ }^{1} \mathrm{H}$ NMR $\left(400 \mathrm{MHz}\right.$ DMSO- $\left.d_{6}\right) \delta 7.92-8.00(\mathrm{~m}, 2 \mathrm{H}, \mathrm{ArH}), 7.84-7.90(\mathrm{~d}$, $J$ 7.6Hz, 2H,ArH), 7.25(m,2H, ArH), 6.94-6.97(d, $J 7.6 \mathrm{~Hz}, 2 \mathrm{H}, \mathrm{ArH}), 3.31-$ 3.34(m, 4H,piperidine $\left.\mathrm{N}-\mathrm{CH}_{2}\right), 1.53-1.71\left(\mathrm{~m}, 6 \mathrm{H}\right.$, piperidine $\left.\mathrm{CH}_{2}\right) ; \mathrm{m} / \mathrm{z}$ 295(M+H). Elemental analysis Calcd. for $\mathrm{C}_{18} \mathrm{H}_{18} \mathrm{~N}_{2} \mathrm{~S}$. C, 73.43; H, 6.16; N, 9.51. Found: C, 73.50; H, 6.20; N, 9.45.

General Procedure for the Preparation of 5a-5f

o-amino phenol (1mmol), aromatic aldehyde $(1.1 \mathrm{mmol})$ and water $(10 \mathrm{~mL})$ were mixed in $25 \mathrm{~mL}$ single neck round bottom flask, and to this Ammonium Nickel Sulphate $(10 \mathrm{~mol} \%)$ was added. The reaction mixture was sonicated at room temperature $\left(25^{\circ} \mathrm{c}\right)$ for the appropriate time (Table 2, entries 26-31), and the progress of reaction was monitored by TLC. After completion of reaction, the mixture was extracted with ethyl acetate $(2 \times 10 \mathrm{~mL})$. The combined organic layer was dried over anhydrous $\mathrm{Na}_{2} \mathrm{SO}_{4}$ and evaporated under reduced pressure; the crude material was purified by column chromatography over silica gel to afford products $5 \mathrm{a}-5 \mathrm{f}$ with high purity.

Selected spectral data

2- phenyl benzoxazole $\mathbf{5 a}$

${ }^{1} \mathrm{H}$ NMR $(400 \mathrm{MHz}$ DMSO- $d$ ) $\delta 8.32(\mathrm{dd}, J 5.6 \mathrm{~Hz}, 2.1 \mathrm{~Hz}, 2 \mathrm{H}, \mathrm{ArH})$, 7.79-7.86(m, $1 \mathrm{H}, \mathrm{ArH}), 7.53-7.67(\mathrm{~m}, 4 \mathrm{H}, \mathrm{ArH}), 7.36-7.44(\mathrm{~m}, 2 \mathrm{H}, \mathrm{ArH}) ; \mathrm{m} / z$ 196(M+H). Elemental analysis Calcd. for $\mathrm{C}_{13} \mathrm{H}_{9} \mathrm{NO}$. C, 79.98; H, 4.65; N, 7.17. Found: C, 79.91; H, 4.70; N, 7.20.

\section{2-(4-methoxyphenyl)benzoxazole $\mathbf{5 b}$}

${ }^{1} \mathrm{H}$ NMR(400MHz DMSO) $\delta 8.22(\mathrm{~d}, J 8.2 \mathrm{~Hz}, 2 \mathrm{H}, \mathrm{ArH}), 7.85(\mathrm{~m}, 1 \mathrm{H}$, $\mathrm{ArH}), 7.61(\mathrm{~m}, 1 \mathrm{H}, \mathrm{ArH}), 7.37-7.42(\mathrm{~m}, 2 \mathrm{H}, \mathrm{ArH}), 7.16(\mathrm{~d}, J 8.2 \mathrm{~Hz}, 2 \mathrm{H}, \mathrm{ArH})$, 3.89(s, 3H, OCH3); $m / z 226(\mathrm{M}+\mathrm{H})$. Elemental analysis Calcd. for $\mathrm{C}_{14} \mathrm{H}_{11} \mathrm{NO}_{2}$. C, 74.65; H, 4.92; N, 6.22. Found: C, 74.70; H, 4.88; N, 6.17.

\section{2-(4-cyanophenyl)benzoxazole $\mathbf{5 c}$}

${ }^{1} \mathrm{H}$ NMR(400MHz DMSO- $d$ ) $\delta 8.32(\mathrm{~d}, J 8.5 \mathrm{~Hz}, 2 \mathrm{H}, \mathrm{ArH}), 7.81-$ 7.97(m,3H, ArH), 7.51(m, 1H, ArH), 7.31-7.49(m, 2H,ArH); m/z 221(M+H). Elemental analysis Calcd. for $\mathrm{C}_{14} \mathrm{H}_{8} \mathrm{~N}_{2} \mathrm{O} . \mathrm{C}, 76.35 ; \mathrm{H}, 3.66 ; \mathrm{N}, 12.72$. Found: C, 76.28; H, 3.70; N, 12.68 . 
2-(3,4- dichlorophenyl)benzoxazole 5d

${ }^{1} \mathrm{H}$ NMR(400MHz DMSO) $\delta 8.37(\mathrm{~d} J 8.1 \mathrm{~Hz}, 1 \mathrm{H}, \mathrm{ArH}), 8.19(\mathrm{dd}, J 8.2 \mathrm{~Hz}$, $1.8 \mathrm{~Hz}, 1 \mathrm{H}, \mathrm{ArH}), 7.72-7.83(\mathrm{~m}, 1 \mathrm{H}, \mathrm{ArH}), 7.62-7.71(\mathrm{~m}, 2 \mathrm{H}, \mathrm{ArH}), 7.40-$ 7.45(m, 2H, ArH); $m / z$ 264(M+H). Elemental analysis Calcd. for $\mathrm{C}_{13} \mathrm{H}_{7} \mathrm{Cl}_{2} \mathrm{NO}$. C, 59.12; H, 2.67; N, 5.30. Found: C, 59.20; H, 2.62; N, 5.24.

6-methyl-2phenyl benzoxazole $\mathbf{5 e}$

${ }^{1} \mathrm{H}$ NMR(400MHz DMSO- $d$ ) $\delta 8.26-8.39(\mathrm{~m}, 2 \mathrm{H}, \mathrm{ArH}), 7.70(\mathrm{~d}, J 8.2 \mathrm{~Hz}$, $1 \mathrm{H}, \mathrm{ArH}), 7.55-7.62(\mathrm{~m}, 3 \mathrm{H}, \mathrm{ArH}), 7.35(\mathrm{~m}, 1 \mathrm{H}, \mathrm{ArH}), 7.09(\mathrm{~m}, 1 \mathrm{H}, \mathrm{ArH}), 2.51(\mathrm{~s}$, $\left.3 \mathrm{H}, \mathrm{CH}_{3}\right) ; \mathrm{m} / z 210(\mathrm{M}+\mathrm{H})$. Elemental analysis Calcd. for $\mathrm{C}_{14} \mathrm{H}_{11} \mathrm{NO}$. C, 80.36; H, 5.30; N, 6.69. Found: C, 80.43; H, 5.26; N, 6.60 .

6-fluoro-2-phenyl benzoxzole $\mathbf{5 f}$

${ }^{1} \mathrm{H}$ NMR(400MHz DMSO) $\delta 8.20-8.28(\mathrm{~m}, 2 \mathrm{H}, \mathrm{ArH}), 7.79(\mathrm{dd}, J 8.8 \mathrm{~Hz}$, $4.9 \mathrm{~Hz}, 1 \mathrm{H}, \mathrm{ArH}), 7.52-7.61(\mathrm{~m}, 3 \mathrm{H}, \mathrm{ArH}), 7.29(\mathrm{dd}, J 8.0,2.3 \mathrm{~Hz}, 1 \mathrm{H}, \mathrm{ArH})$, 7.11(m, $1 \mathrm{H}, \mathrm{ArH}) ; \mathrm{m} / \mathrm{z} 214(\mathrm{M}+\mathrm{H})$. Elemental analysis Calcd. for $\mathrm{C}_{13} \mathrm{H}_{8} \mathrm{FNO}$. C, 73.23; H, 3.78; N, 6.57. Found: C, 73.20; H, 3.83; N, 6.50.

\section{RESULT AND DISCUSSION}

We developed an ecofriendly, one pot Ammonium Nickel Sulfate catalyzed synthesis of 2-aryl substituted benzimidazole, benzoxazole and benzothiazole in water.

2-(4-methoxy phenyl)benzimidazole (3b)(scheme 1) was selected as a proto type compound to optimize the reaction condition (Table 1) from o-phenylene diamine (1) and anisaldehyde (2). We first conducted the reaction of $\mathbf{1}$ ( 1 equivalent) and $\mathbf{2}$ (1.1 equivalent) in the presence of Ammonium Nickel Sulfate in water at different temperature (Table 1 entries 10 to 12 ). The yield increases from $30 \%$ to $50 \%$ up to $80^{\circ} \mathrm{C}$ in 8 hours. With the aim to reduce reaction time and temperature, we move to non-conventional energy source i.e. ultrasound energy, so we carried the reaction under ultrasound, to afford the corresponding 2-(4-methoxy phenyl)benzimidazole (3b) in $85 \%$ yield ( Table 1 entry 13 )in much shorter time at room temperature. To know the effect of solvent, We kept the catalyst constant and used different solvent $\mathrm{CH}_{2} \mathrm{Cl}_{2}$, $\mathrm{CH}_{3} \mathrm{CN}, \mathrm{THF}, \mathrm{CHCl}_{3}$, DMF, EtOH ,DMSO and Dioxane but all afford a very low yield ( Table 1 entries 1-8). These indicate that water is the best solvent for the synthesis of $\mathbf{3 b}$; it may be due to solubility of catalyst, varying the amount of catalyst did not improve the yield (Table 1 entries 14,15 )
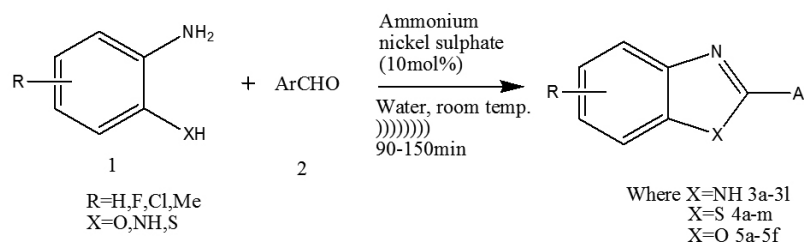

Scheme 1

With optimal condition in hand, we reacted various substituted o-phenylene diamine $\mathbf{1}$ and aromatic aldehyde $\mathbf{2}$ to give the corresponding substituted 2 -aryl benzimidazole product 3a to $\mathbf{3 l}$ (Table 2 entries 1-12). A wide variety of aldehydes were used, which have both electron donating and electron withdrawing substituents along with substituted o-phenylene diamine. The method is also suitable for heteroaromatic aldehyde.

Table 1. Solvent effect on the reaction of anisaldehyde and o-phenylene diamine in presence of ammonium nickel sulphate.

\begin{tabular}{|c|c|c|c|c|c|c|}
\hline Entry & solvent & catalyst & Temp. $/{ }^{\circ} \mathrm{C}$ & Time/min & Product $^{\mathrm{a}}$ & Yield $(\%)^{\mathrm{b}}$ \\
\hline 1 & $\mathrm{CH}_{2} \mathrm{Cl}_{2}$ & 10 & $\mathrm{RT}^{*}$ & 120 & $3 b$ & Traces \\
\hline 2 & $\mathrm{CHCl}_{3}$ & 10 & $\mathrm{RT}^{*}$ & 120 & $3 b$ & Traces \\
\hline 3 & DMSO & 10 & $\mathrm{RT}^{*}$ & 120 & $3 b$ & 45 \\
\hline 4 & DMF & 10 & $\mathrm{RT}^{*}$ & 120 & $3 b$ & 45 \\
\hline 5 & $\mathrm{EtOH}$ & 10 & $\mathrm{RT}^{*}$ & 120 & $3 b$ & 30 \\
\hline 6 & $\mathrm{CH}_{3} \mathrm{CN}$ & 10 & $\mathrm{RT}^{*}$ & 120 & $3 b$ & 40 \\
\hline 7 & Dioxane & 10 & $\mathrm{RT}^{*}$ & 120 & $3 b$ & 38 \\
\hline 8 & THF & 10 & $\mathrm{RT}^{*}$ & 120 & $3 b$ & 36 \\
\hline 9 & $\mathrm{H}_{2} \mathrm{O}$ & 10 & RT & 8Hours & $3 b$ & Traces \\
\hline 10 & $\mathrm{H}_{2} \mathrm{O}$ & 10 & 40 & 8 Hours & $3 b$ & 25 \\
\hline 11 & $\mathrm{H}_{2} \mathrm{O}$ & 10 & 60 & 8 Hours & $3 b$ & 40 \\
\hline 12 & $\mathrm{H}_{2} \mathrm{O}$ & 10 & 80 & 8 Hours & $3 b$ & 50 \\
\hline 13 & $\mathrm{H}_{2} \mathrm{O}$ & 10 & $\mathrm{RT}^{*}$ & 120 & $3 b$ & 85 \\
\hline 14 & $\mathrm{H}_{2} \mathrm{O}$ & 05 & $\mathrm{RT}^{*}$ & 120 & $3 b$ & 65 \\
\hline 15 & $\mathrm{H}_{2} \mathrm{O}$ & 15 & $\mathrm{RT}^{*}$ & 120 & $3 b$ & 85 \\
\hline
\end{tabular}

* Under ultra-sonication.

${ }^{\mathrm{a}} 1 \mathrm{mmole}$ of o-phenylene diamine, $1.1 \mathrm{mmol}$ anisaldehyde, $10 \mathrm{~mol} \%$ catalyst, solvent, room temperature. ${ }^{\mathrm{b}}$ Isolated yield based on starting o-phenylene diamine.

The scope of above procedure was expanded to the synthesis of2aryl benzoxazole from o-amino phenol and 2-aryl benzothiazole from o-aminothiophenol, the results are summarized in Table 2. Aromatic aldehyde with electron donating as well as electron withdrawing groups participated in the reaction, the nature and position of substituent on the aryl ring does not make much difference in reactivity. The structures of the products were confirmed by ${ }^{1} \mathrm{H}$ NMR, mass spectra. 
J. Chil. Chem. Soc., 59, No 1 (2014)

Table 2. Ammonium nickel sulphate $\left[\left(\mathrm{NH}_{4}\right)_{2} \mathrm{SO}_{4} \cdot \mathrm{NiSO}_{4} \cdot 6 \mathrm{H}_{2} \mathrm{O}\right]$ catalyzed of 2-arylbenzimidazole, 2-arylbenzothiazole and 2-arylbenzoxazole under ultrasonication in water

Standard condition: 1mmole of o-phenylene diamine or o-aminothiophenol or o-aminophenol, 1.1mmol ArCHO, 10mol\% catalyst(ammonium nickel sulphate), water, room temperature. Isolated yield based on starting o-phenylene diamine or o-aminothiophenol or o-aminophenol

\begin{tabular}{|c|c|c|c|c|c|c|c|c|}
\hline \multirow{2}{*}{ entry } & \multirow{2}{*}{$\mathrm{R}$} & \multirow{2}{*}{$\mathrm{Ar}$} & \multirow{2}{*}{$X$} & \multirow{2}{*}{ product } & \multirow{2}{*}{ Time(min) } & \multirow{2}{*}{ yield } & \multicolumn{2}{|c|}{ M.Pt ${ }^{\circ} \mathrm{C}$} \\
\hline & & & & & & & observed & reported \\
\hline 1 & $\mathrm{H}$ & & $\mathrm{N}$ & $3 a$ & 135 & 80 & $287-288$ & $292^{37}$ \\
\hline 2 & $\mathrm{H}$ & & $\mathrm{N}$ & $3 b$ & 120 & 85 & $228-230$ & $226^{37}$ \\
\hline 3 & $\mathrm{H}$ & & $\mathrm{N}$ & $3 c$ & 110 & 89 & $288-291$ & $294^{37}$ \\
\hline 4 & $5-\mathrm{Cl}$ & & $\mathrm{N}$ & $3 d$ & 140 & 86 & $276-279$ & $278-279^{40}$ \\
\hline 5 & $5-\mathrm{Cl}$ & & $\mathrm{N}$ & $3 e$ & 105 & 87 & $258-259$ & $260-261^{40}$ \\
\hline 6 & $5-\mathrm{Cl}$ & & $\mathrm{N}$ & $3 \mathrm{f}$ & 150 & 85 & $311-313$ & $310-312^{43}$ \\
\hline 7 & $\mathrm{H}$ & & $\mathrm{N}$ & $3 g$ & 95 & 85 & $200-202$ & $204-206^{38}$ \\
\hline 8 & $\mathrm{H}$ & & $\mathrm{N}$ & $3 \mathrm{~h}$ & 110 & 88 & $243-246$ & $245-248^{42}$ \\
\hline 9 & $\mathrm{H}$ & & $\mathrm{N}$ & $3 \mathrm{i}$ & 145 & 83 & $175-177$ & $178-179^{39}$ \\
\hline 10 & $\mathrm{H}$ & & $\mathrm{N}$ & $3 \mathrm{j}$ & 120 & 87 & $219-221$ & $220-222^{39}$ \\
\hline 11 & $\mathrm{H}$ & & $\mathrm{N}$ & $3 \mathrm{k}$ & 125 & 88 & $230-231$ & $234^{37}$ \\
\hline 12 & $\mathrm{H}$ & & $\mathrm{N}$ & 31 & 110 & 90 & $308-310$ & $316^{37}$ \\
\hline 13 & $\mathrm{H}$ & & $\mathrm{S}$ & $4 a$ & 90 & 91 & $110-112$ & $113-114$ \\
\hline 14 & $\mathrm{H}$ & & $\mathrm{S}$ & $4 b$ & 95 & 90 & $121-124$ & $120-122$ \\
\hline 15 & $\mathrm{H}$ & & $\mathrm{S}$ & $4 c$ & 85 & 86 & $224-227$ & $226-228$ \\
\hline
\end{tabular}


J. Chil. Chem. Soc., 59, Nº 1 (2014)

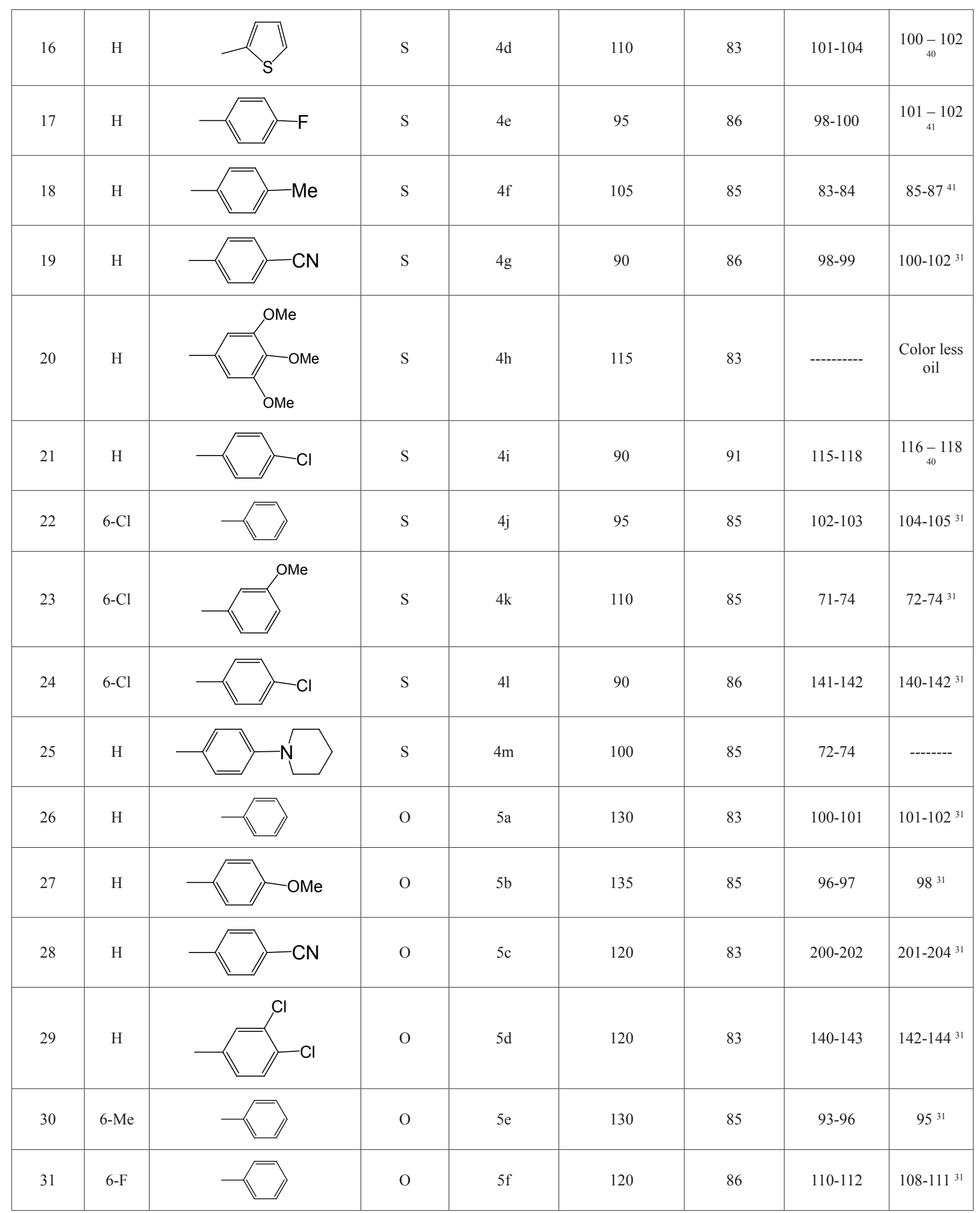




\section{CONCLUSIONS}

Ammonium Nickel Sulphate as a catalyst provides cheap, safe and environment friendly procedure for the synthesis of 2-aryl substituted benzimidazole, benzoxazole and benzothiazole derivatives from various aromatic aldehydes with o-phenylene diamine, o-amino phenol and o-amino thiophenol. The advantages offered by this method are operational simplicity, good yield of product.

\section{ACKNOWLEDGMENTS}

The authors are grateful to the Head, Department of Chemistry, Vinayakrao Patil Mahavidyalaya, Vaijapur for providing the laboratory facility and Director, SAIF, Chandigarh for providing spectral analysis of synthesized compounds.

\section{REFERENCES}

1. D. A. Horton, G. T. Bourne, M. L. Smythe, Chem.Rev., 2003, 103, 893.

2. A. A. Weekes, A. D. Westwell, Curr.Med.Chem., 2009, 16, 2430.

3. B. Gong, F. Hong, C. Kohm, L. Bonham, P. Klein, Bioorg.Med.Chem. Lett.2004,14,1455.

4. S. M. Sodhi, N. Singh, A. Kumar, O. Lozach, L. Meijer, Bioorg.Med. Chem., 2006, 14, 3758.

5. J. Vinsona, K. Cermakova, A. Tomeckova, M. Ceckova, J. Jampilek, P. Cermak, J. Kunes, M. Dolezal, F. Staud, Bioorg. Med.Chem., 2006, 14, 5850.

6. I. O. Yildiz, I. Yalcin, E. Akisener, N. Ueartark, Eur.J.Med.Chem., 2004, $39,291$.

7. X. Song, B. S. Vig, P. L. Lorenzi, J. C. Drach, L. B. Townsend, G. L. Amidon, J.Med.Chem., 2005, 48, 1274.

8. M. Yamoto, J.Pharm.Soc.Jpn., 1992, 112, 81.

9. D. Kumar, M. R. Jacob, M. B. Rcynold, S. M. Karvin, Bioorg.Med.Chem., 2002, 10, 3997.

10. A. Benazzouz, T. Boraud, P. Dubedat, A. Bioreau, J. M. Stutzmann, C. Gross, Eur.J.Pharmacol., 1995, 284, 299.

11. A. R. Katritzky, C. A. Ramsden, E. F. V. Scriven, R. J. K. Taylor, Comprehensive Heterocyclic Chemistry III, Vol. 3; ed. 1; Elsevier : London 2008

12. E. Alcalde, I. Dinares, L. Perez-Garcia, T. Roca, T.; Synthesis, 1992, 395.

13. R. S. Pottorf, N. K. Chadha, M. Katkevics, V. Ozola, E. Suna, H. Ghane, T. Regberg, M. R. Player, Tetrahedron Lett., 2003, 44, 175.

14. R. N. Nadaf, S. A. Siddiqui, T. Daniel, R. J. Lahoti, K. V. Srinivasan, J. Mol. Catal. A: Chem., 2004, 214, 155.

15. G. L. Jenkins, A. M. Knevel, C. S. Davis, J. Org. Chem., 1961, $26,274$.

16. M. Doise, F. Dennin, D. Blondeau, H. Sliwa, Tetrahetron Lett., 1990, 31, 1155.
17. D. Villemin, M. Hammadi, B. Martin, Synthetic Commun., 1996, 26, 2895.

18. A. K. Chakraborti, S. Rudrawar, G. Kaur, L. Sharma, Synlett., 2004, 1533.

19. A. Hari, C. Karan, W. C. Rodridues, B. L. Miller, J. Org. Chem., 2001, 66, 991.

20. P. L. Beaulieu, B. Hache, E. Von Moss, Synthesis, 2003, 1683.

21. M. Curini, F. Epifano, F. Montanari, O. Rosati, S. Taccone, Synlett., 2004, 1832.

22. P. Gogoi, D. Konwar, Tetrahedron Lett., 2006, 47, 79.

23. R. Trivedi, S. K. De, R. A. Gibbs, J. Mol. Catal. A: Chem., 2006, 245, 8 .

24. V. G. Navarrete, D. H. Moreno, C. F. Aguirre, R. I. Leon, M. R. Villalobos, M. O. Munoz, S. S. Estrada, Bioorg. Med. Chem. Lett., 2006, 16, 4169.

25. G. V. Boyd, A. R. Katritzky, C. W. Rees, K. T. Potts, Comprehensive Heterocyclic Chemistry, Pregamon Press: New York, 1984, 177.

26. X. Z. Bu, Z. S. Huang, M. Zhang, L. Ma, G. W. Xiao, L. Q. Gu, Tetrahedron Lett., 2001, 42, 5737.

27. B. M. Bhaval, S. P. Mayabhate, A. P. Likhite, A. R. Deshmukh, Synthetic Commun., 1995, 25, 3315.

28. Y. Riadi, R. Mamouni, R. Azzalou, M. El Haddad, S. Routier, G. Guillaumet, S. Lazar, Tetrahedron Lett., 2011, 52, 3492.

29. H. M. Bachhav, S. B. Bhagat, V. N. Telvekar, Tetrahedron Lett. ,2011, 52, 5697.

30. A. J. Blacker, M. M. Farah, M. L. Hall, S. P. Marsden, O. Saidi, J. M. Williams, Org.Lett., 2009, 11, 2039.

31. S. S. Pawar, D. V. Dekhane, M. S. Shingare, S. N. Thore, Australian J. Chem., 2008, 61, 905.

32. S. S. Pawar, D. V. Dekhane, M. S. Shingare, S. N. Thore, Chinese Chemical Letters , 2008, 19, 1055.

33. T. J.Mason, J. P. Lorimer, Sonochemistry: Theory, Application and Uses of Ultrasound in Chemistry; John Wiley and Son: New York, 1988.

34. K. S. Suslick, Ultrasound, its Chemical, Physical and Biological Effects; VCH: Weinheim, 1988.

35. A. Gaplovsky, M. Gaplovsky, S. Toma, J. L. Luche, J. Org. Chem., 2000, 65,8444 .

36. R. R. Deshmukh, R. Rajagopal, K. V. Srinivasan, Chem. Commun., 2001, 1544.

37. A. Ben-Alloum, K. Bougrin, M. Soufiaoui, Tetrahedron Lett., 2003, 44, 5935.

38. M. R. DeLuca, S. M. Kerwin, Tetrahedron, 1997, 53, 457.

39. S. E. López, J. Restrepo, B. Pérez, S. Ortiz, J. Salazar, Bull. Korean Chem. Soc., 2009, 30, 7.

40. K. S. Niralwad, B. B. Shingate, M. S. Shingare, Bull. Korean Chem. Soc., 2010, 31, 981

41. D. Azarifar, B. Maleki, M. Setayeshnazar, Phosphorus, Sulfur Silicon Relat. Elem., 2009, 184, 2097.

42. H. J. Myung, M. P. Jung, C. L. Ihl-Young, A. Miya, J. Heterocyclic Chem., 